\title{
NUMERICAL APPROACH TO THE EVALUATION OF ATMOSPHERIC POLLUTION BY COMBUSTION PRODUCTS OF SOLID FUELS
}

\author{
Dmitry Gvozdyakov ${ }^{1, ~}{ }^{*}$, Vladimir Gubin ${ }^{1}$, Svetlana Shvab $^{1}$, and Anton Tanishev ${ }^{1}$ \\ ${ }^{1}$ National Research Tomsk Polytechnic University, 634050 Tomsk, Russia
}

\begin{abstract}
This research presents the results of mathematical modeling of acid rain formation and its fallout in the areas adjacent to the site of the energy complex. Four thermal periods that are typical for regions of sharply continental climate has been considered. The influence of convective parameters on the formation mechanism of atmospheric precipitation has been estimated. The contribution of solar energy flux to the research process has been analyzed.
\end{abstract}

\section{Introduction}

Nowadays one of the main sources of emission combustion products of solid fuels in atmospheric is chemical and energy complexes [1]. Every year emissions of toxic compounds, i.e. $\mathrm{SO}_{3}$, in environment are increase. [2]. The interaction (condensation on the surface of microscopic particles) of the sulfuric anhydride with the components of air can lead to formation of acid rain [1]. Researches [3] are shown that acid formation cause considerable damage not only in the acid rain, actually. According to previous research [4] - with the increase (or decreasing) in the speed of air masses can change the mass flow rate of condensation $\mathrm{SO}_{3}$ on the droplet surface. Experience to solve problems [5-6] shows the ability of numerical approach in the processes of transformation and distribution in airspace of combustion of natural fuel thermal power plants produce.

The purpose of this research is the numerical approach to the study of the process of formation and distribution of the sedimentation of acid rain in the atmosphere adjacent to the thermal power plant, on the surface of condensation nuclei, with the main significant factors: unsteady heat and mass transfer; phase transformations; solar radiation; the speed of movement of air masses; temperature; drops of flight dynamics.

We used an iterative algorithm developed for solving nonlinear heat conduction problems in a local heat exposure [7] method of finite differences.

According to [8], the influence of non-sphericity coefficient drops characterized nonsphericity $\mathrm{G}$, as determined ratio of the surface area of a real drop in the area of similar size sphere.

* Corresponding author: Dim2003@tpu.ru 


\section{Mathematical problem}

Heat equations drops and movement within the accepted physical model of the test process is as follows:

$$
\left.\begin{array}{c}
\rho_{1} \cdot C_{P} \cdot \frac{\partial T_{1}}{\partial t}=\lambda\left(\frac{\partial^{2} T_{1}}{\partial r^{2}}+\frac{2}{r} \cdot \frac{\partial T_{1}}{\partial r}\right) \\
\frac{d U_{X}}{d t}=\frac{3 \cdot G \cdot C_{D} \cdot \rho_{1} \cdot\left(U_{X}-U_{0}\right) \cdot\left(U_{X}-U_{0}\right)}{8 \cdot \pi \cdot \delta \cdot \rho_{2}} \\
\frac{d V_{Y}}{d t}=g+\frac{3 \cdot G \cdot C_{D} \cdot \rho_{1} \cdot\left(V_{Y}-V_{0}\right) \cdot\left(V_{Y}-V_{0}\right)}{8 \cdot \pi \cdot \delta \cdot \rho_{2}}
\end{array}\right\}
$$

where $\rho_{1,2}$ - density, $\mathrm{kg} / \mathrm{m}^{3} ; C_{P}$ - isobaric heat capacity, $J /(\mathrm{kg} \cdot \mathrm{K}) ; \lambda$ - coefficient of thermal conductivity, $W /(m \cdot K) ; T$ - temperature, $K ; t$ - time, $s ; V_{X}, V_{Y}$ - drop velocity in the direction of the axes $x$ and $y$, respectively, $\mathrm{m} / \mathrm{s} ; C_{D}$ - drag coefficient; $g$ - acceleration of gravity, $\mathrm{m} / \mathrm{s}^{2} ; U_{0}, V_{0}$ - velocity components ambient, $\mathrm{m} / \mathrm{s}$.

The initial and boundary conditions are:

$$
\begin{gathered}
t=0: \quad T=T_{0}, \quad 0 \leq r \leq \delta \\
r=0:-\lambda_{1} \cdot \frac{\partial T_{1}}{\partial r}+Q \cdot\left(W_{K}^{3}+W_{K}^{4}\right)-P=-\lambda_{2} \cdot \frac{\partial T_{2}}{\partial r}, \quad T_{1}=T_{2} ; \quad r=\frac{\delta}{2}: \quad \frac{\partial T_{1}}{\partial r}=0,
\end{gathered}
$$

where $T_{1}, T_{2}$ - the temperature drops and a gaseous medium, respectively, $K ; W_{k}^{3} W_{k}^{4}-$ mass condensation rate, $\mathrm{kg} /\left(\mathrm{m}^{2} \cdot \mathrm{s}\right) ; Q$ - the heat of reaction, $\mathrm{J} / \mathrm{kg} ; \delta$ and $r$ - the characteristic dimensions of the drops, $m ; P$ - density of solar energy flux, $W / m^{2}$.

Two thermal periods are analyzed, typical for regions with sharply continental climate (i.e., Siberia). The value of the solar energy flux density was assumed to be the value of the solar constant. Below in fig. 1 illustrates the results of numerical studies in the form of temperature drop of the surface from a distance (blue dashed lines correspond to the temperature of the air).

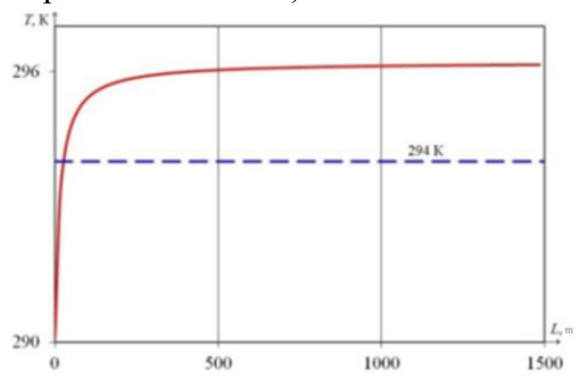

a)

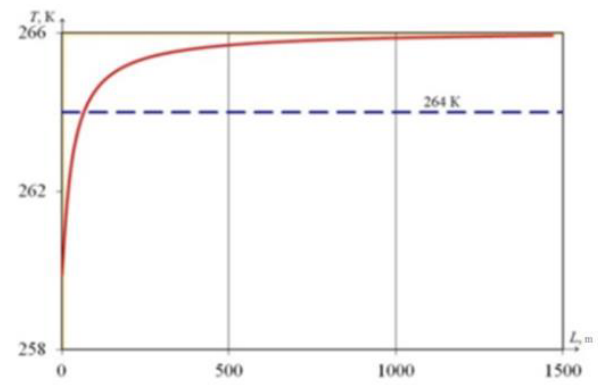

b)

Fig. 1. Temperature fields of drop at the time $1800 \mathrm{~s}$ at the wind speed of $5 \mathrm{~m} / \mathrm{s}$ : a) in the summer; b) in the winter.

Secondly, the influence of the wind speed on warming formed drops is substantially. The difference between the temperature of sulfuric acid droplets formed surfaces in the airspace, adjacent to the thermal power plant, and the environment is $0.5 \ldots 1.2 \mathrm{~K}$. Numerical evaluation of the impact of the speed of movement of air masses (fig. 1) is consistent with studies [8,9]. Below in fig. 2 are shown the results of mathematical modeling. 


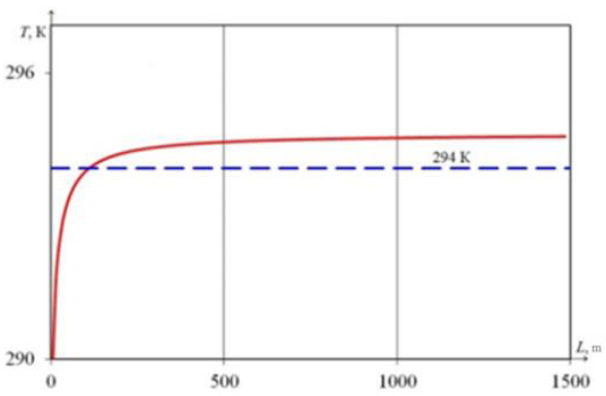

a)

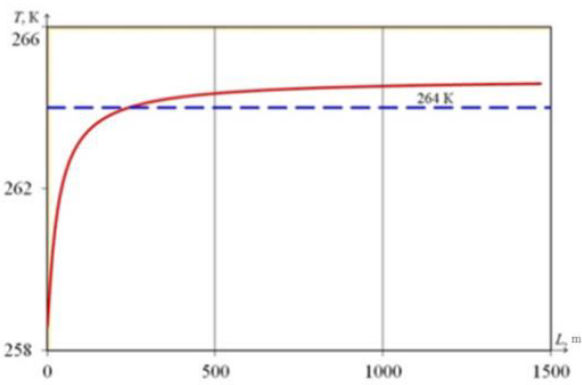

b)

Fig. 2. Temperature fields of drop at the time $1800 \mathrm{~s}$ when at the wind speed of $10 \mathrm{~m} / \mathrm{s}$ : a) in the summer; b) in the winter.

Table 1 shows a comparison of the results of research [9] and the present study. Comparison of the numerical values of mathematical modeling of the formation of acid formation on the surface of condensation nuclei is performed at the wind speed of $5 \mathrm{~m} / \mathrm{s}$ and ambient temperature of $294 \mathrm{~K}$.

Table 1. Dimensions drops of sulfuric acid with different mechanisms of formation of acid rain.

\begin{tabular}{|c|c|c|}
\hline \multirow{2}{*}{$t, s$} & {$[9]$} & $\begin{array}{c}\text { Condensation of } \mathrm{SO}_{3} \& \mathrm{H}_{2} \mathrm{O} \text { with } \\
\text { considering } P\end{array}$ \\
\cline { 2 - 3 } & $\delta_{c h} \cdot 10^{3}, m$ & $\delta_{c h} \cdot 10^{3}, m$ \\
\hline 0 & \multicolumn{2}{|c|}{$\delta_{0}=5.0 \cdot 10^{6}, m$} \\
\hline 600 & 0.960 & 0.923 \\
\hline 1800 & 1.616 & 1.553 \\
\hline
\end{tabular}

Here $\delta_{0}$ is the size of condensation nuclei in the initial time, $m$; $\delta_{c h}-$ is particle size formed at time $t$.

\section{Conclusion}

Evaluation of the numerical parameters of acid precipitation leads to the conclusion that cocondensation of vapor of sulfuric anhydride and steam based on the solar energy flux significantly (up $4 \%$ ) slows the process of formation of sedimentary deposits. It should be noted that after $600 \mathrm{~s}$ precipitation with a typical size of $1 \mathrm{~mm}$ are formed.

\section{Acknowledgments}

The research was realized with financial support of Minobrnauki of Russia in framework of FTP "Research and development in prior direction of scientific-technological complex of Russia in 2014-2020 years”, unique R\&D identifier RFMEFI58114X0001.

\section{References}

1. Y. Izrael, I.Nazarov, A.Pressman, Kislotnye dozhdi (Acid rain. Gidrometeoizdat. Leningrad, 1989)

2. E. Volkov, E. Gavrilov, F. Dugih, Flue pipe TPP and NPP (Energoatomizdat. Moscow, 1987)

3. D. Gvozdyakov, V. Gubin, Butlerov Communications 37, 10 (2013) 
4. M.Thomas, Impact of air pollution on plants. Geneva (1962)

5. D. Gvozdyakov, V. Gubin, Chem. Pet. Eng. 51 (2015)

6. D. Gvozdyakov, V. Gubin, EPG Web Conf. 82, Article Number 01026 (2015)

7. G. Kuznetsov, M. Sheremet, Russ. Microelectron. 37, 2 (2008)

8. V. Terekhov, M. Pakhomov, Heat and mass transfer and hydrodynamics in gas-drop flows (NSTU, Novosibirsk, 2008)

9. D. Gvozdyakov, V. Gubin, Chem. Pet. Eng. 3 (2015) 